The Astrophysical Journal, 504:442-449, 1998 September 1

(C) 1998. The American Astronomical Society. All rights reserved. Printed in U.S.A.

\title{
IRROTATIONAL BINARY NEUTRON STARS IN QUASI-EQUILIBRIUM IN GENERAL RELATIVITY
}

\author{
Saul A. Teukolsky ${ }^{1}$ \\ Center for Radiophysics and Space Research, Cornell University, Ithaca, NY 14853 \\ Received 1998 March 9; accepted 1998 April 7
}

\begin{abstract}
Neutron stars in binary orbit emit gravitational waves and spiral slowly together. During this inspiral, they are expected to have very little vorticity. It is in fact a good approximation to treat the system as having zero vorticity, i.e., as irrotational. Because the orbital period is much shorter than the radiation reaction timescale, it is also an excellent approximation to treat the system as evolving through a sequence of equilibrium states, in each of which the gravitational radiation is neglected. In Newtonian gravity, one can simplify the hydrodynamic equations considerably for an equilibrium irrotational binary by introducing a velocity potential. The equations reduce to a Poisson-like equation for the potential, and a Bernoulli-type integral for the density. We show that a similar simplification can be carried out in general relativity. The resulting equations are much easier to solve than other formulations of the problem.
\end{abstract}

Subject headings: binaries: close - gravitation — hydrodynamics - relativity - stars: neutron stars: rotation

\section{INTRODUCTION}

Ever since the discovery of the first neutron star binary by Hulse \& Taylor (1975), we have known that these systems exist and will undergo orbital decay via gravitational wave emission. The inspiral and coalescence of binary neutron stars is one of the primary targets for gravitational wave detectors now under construction, such as LIGO, VIRGO, and GEO (see, e.g., Abramovici et al. 1992; Thorne 1994). And the coalescence of binary neutron stars is the basis for several models for gamma-ray bursters (Paczyński 1986; Eichler et al. 1989; Narayan, Paczyński, \& Piran 1992). Clearly, a theoretical understanding of coalescing binary neutron stars is an important astrophysical problem.

Analyzing coalescing neutron stars requires the full machinery of general relativity. While some issues can be addressed with Newtonian or post-Newtonian gravity, such as the gravitational waveform at large separation, others cannot even be posed in these weak-field limits. For example, consider the recent controversial claim by Wilson, Mathews, and Marronetti (Wilson \& Mathews 1995; Wilson, Mathews, \& Marronetti 1996; Mathews, Marronetti, \& Wilson 1997) that massive neutron stars collapse to black holes before they merge. Gravitational collapse of a star in equilibrium is a consequence of the nonlinear nature of Einstein's equations; it cannot be treated correctly with Newtonian gravity. If neutron star binaries do in fact collapse before they merge, there are enormous implications for gravitational wave detection: The entire detection strategy is based on having accurate templates for the waveforms, and current templates predict relatively high sensitivity because without collapse there are many orbital periods before coalescence. And of course if the neutron stars collapse, then their final coalescence cannot be a source of gamma rays.

There are other aspects of the inspiral problem that require a fully relativistic treatment. For example, if the stars do not collapse before merger, their combined mass is likely to be greater than the maximum mass of a cold, rotating neutron star. Then the merged remnant must ultimately collapse to a black hole. But it is not clear whether this collapse occurs immediately on a dynamical timescale, or whether thermal pressure from shock heating will be sufficient to hold the star up for a while. In this case, collapse will occur on a neutrino-dissipation timescale. We also do not know how much angular momentum the final remnant will have. What will the fate of the system be if the angular momentum exceeds the maximum allowed value for a Kerr black hole? Will the excess angular momentum be radiated away gravitationally, or will it be ejected in a circumstellar ring of matter, possibly leading to planet formation? All these questions have observational implications that can be addressed only by fully relativistic simulations.

A number of groups have undertaken the construction of general relativistic codes that can treat the binary problem. One needs a code that solves Einstein's equations in three spatial dimensions plus time, and simultaneously solves the equations of relativistic hydrodynamics. This is a formidable challenge, and not surprisingly various approximations to the full problem have been proposed. As we have argued above, it seems important not to give up the strong-field aspects of the problem by using Newtonian or post-Newtonian gravity. Instead, we will focus on two approximations that are likely to be well satisfied for real neutron star binaries, at least up until the orbit becomes unstable and the stars finally plunge together: quasiequilibrium and irrotational flow.

In Newtonian gravity, one can find exact equilibrium states for a binary neutron star system. In general relativity, a binary loses energy by gravitational wave emission and the stars spiral ever closer together. However, the timescale on which the orbit changes is much longer than the orbital period, at least until the orbit becomes unstable at the innermost stable circular orbit. We can therefore approximate the evolution as proceeding through a succession of equilibrium states of decreasing separation, in each of which gravitational radiation can be neglected. The rate of progression along the sequence is deter-

\footnotetext{
${ }^{1}$ Also Departments of Physics and Astronomy, Cornell University.
} 
mined by the rate of gravitational wave emission, but the structure at each point along the sequence can be calculated to excellent approximation ignoring the radiation.

Relativistic quasi-equilibrium neutron star binaries have been constructed by Baumgarte et al. (1997a, 1997b, 1997c). For Einstein's equations they used the approximation scheme of Wilson and Mathews (Wilson \& Mathews 1989; Wilson 1990; Wilson \& Mathews 1995), which is a consistent way of neglecting small gravitational radiation terms in Einstein's equations. This scheme has been calibrated by Cook, Shapiro, \& Teukolsky (1996) on rapidly rotating single neutron stars, where it gives excellent results. Wilson and Mathews evolve the binary system by solving the full dynamical equations for the matter in the instantaneous background metric, and then updating the metric quantities at each time step by re-solving the approximate Einstein's equations. Baumgarte et al., on the other hand, take advantage of the quasi-equilibrium approximation for the matter equations too: they reduce the matter equations to a Bernoulli integral, which can be solved much more accurately and conveniently than the original equations of motion.

Using this approach Baumgarte et al. (1997d) studied the stability of binary neutron stars and found no evidence for the instability reported by Wilson and Mathews, even though they were using a very similar approximation scheme. One possible reason for the difference is that the models of Baumgarte et al. are corotating, that is, are locked in synchronous orbit. The models of Wilson and Mathews, by contrast, have very little intrinsic spin. It is possible in principle that the higher spin rate of the corotating models suppresses the instability (Mathews, Marronetti, \& Wilson 1997). As we shall see, the irrotational approximation allows the construction of models almost as easily as the corotating assumption does. However, the irrotational models are essentially nonspinning and should allow one to settle the collapse question definitively.

Realistic neutron stars are in fact unlikely to be corotating; the viscosity is probably too low to synchronize the spin and orbital angular velocities (Bildsten \& Cutler 1992; Kochanek 1992; Lai 1994). Instead, the stars are likely to have very low intrinsic spins compared with the orbital frequency, and to good approximation we can assume they are irrotational (zero vorticity as seen from the inertial frame). Thus, besides being of use in determining whether the Wilson-Mathews instability is real or not, the irrotational approximation will produce models that are close to those expected actually to occur.

Ultimately, even if neutron star binaries do not collapse prematurely to black holes, they will reach an instability corresponding to the innermost stable circular orbit. The subsequent plunge and merger cannot be treated by the quasiequilibrium approximation. However, models constructed with the approximations in this paper should provide excellent initial data for the fully dynamical codes required to treat the final plunge. In fact, because of the extreme computational requirements of dynamical codes, it is unlikely that they will be able to follow the evolution from large separation at all. Realistic initial data such as provided by the methods here will be crucial.

\section{IRROTATIONAL BINARIES IN NEWTONIAN GRAVITY}

Exact irrotational binaries in Newtonian gravity were first constructed by Bonazzola et al. (1992). They used the following method. Start with the equation of motion for a fluid in a uniformly rotating frame of reference:

$$
\frac{\partial v}{\partial t^{\prime}}+(v \cdot \nabla) v+2 \boldsymbol{\Omega} \times \boldsymbol{v}+\mathbf{\Omega} \times(\boldsymbol{\Omega} \times \boldsymbol{r})=-\frac{1}{\rho_{0}} \nabla \boldsymbol{P}-\nabla \phi .
$$

Here $\Omega$ is the constant orbital angular velocity, $P$ is the pressure, $\rho_{0}$ the density, and $\phi$ the Newtonian potential. The origin of the coordinates is at the center of mass of the binary system. The velocity $u$ in the inertial frame is related to the velocity in the rotating frame by

$$
\boldsymbol{u}=\boldsymbol{\Omega} \times \boldsymbol{r}+\boldsymbol{v} .
$$

For later comparison with the relativistic case, note that the time derivatives in the rotating and inertial frames are related by

$$
\frac{\partial}{\partial t^{\prime}}=\frac{\partial}{\partial t}+\mathscr{L}_{w}
$$

where $\boldsymbol{w}=\boldsymbol{\Omega} \times \boldsymbol{r}$ is the orbital velocity and $\mathscr{L}$ denotes the Lie derivative.

Now assume stationarity in the rotating frame: $\partial / \partial t^{\prime} \rightarrow 0$. Then equation (1) can be rewritten as

$$
\nabla\left(\frac{1}{2} v^{2}+h_{\mathrm{N}}+\phi+\phi_{c}\right)+[(\nabla \times v)+2 \Omega] \times v=0 .
$$

Here

$$
h_{\mathrm{N}}=\int \frac{d P}{\rho_{0}}=u+\frac{P}{\rho_{0}}
$$

is the Newtonian enthalpy per unit mass, $u$ is the internal energy per unit mass, and

$$
\phi_{c}=-\frac{1}{2}(\boldsymbol{\Omega} \times \boldsymbol{r})^{2}
$$

is the centrifugal potential. Equation (4) can also be written in terms of the inertial velocity:

$$
\nabla\left(\frac{1}{2} u^{2}-(\boldsymbol{\Omega} \times \boldsymbol{r}) \cdot \boldsymbol{u}+h_{\mathrm{N}}+\phi\right)+(\nabla \times \boldsymbol{u}) \times(\boldsymbol{u}-\boldsymbol{\Omega} \times \boldsymbol{r})=0 .
$$

The continuity equation, with $\partial / \partial t^{\prime} \rightarrow 0$, becomes

$$
\boldsymbol{\nabla} \cdot \boldsymbol{v}=-\boldsymbol{v} \cdot \frac{\nabla \rho_{0}}{\rho_{0}}
$$


or

$$
\boldsymbol{\nabla} \cdot \boldsymbol{u}=-(\boldsymbol{u}-\boldsymbol{\Omega} \times \boldsymbol{r}) \cdot \frac{\nabla \rho_{0}}{\rho_{0}} .
$$

The corotating case corresponds to $v=0$. Equation (8) is trivially satisfied, while equation (4) is just the Bernoulli equation:

$$
h_{\mathrm{N}}+\phi+\phi_{c}=\text { constant } .
$$

The irrotational case corresponds to no vorticity in the inertial frame, $\nabla \times \boldsymbol{u}=0$. This implies that $\boldsymbol{u}$ is given by a velocity potential,

$$
\boldsymbol{u}=\nabla \psi
$$

Equations (7) and (9) become

$$
\begin{gathered}
\frac{1}{2}(\nabla \psi)^{2}-(\boldsymbol{\Omega} \times \boldsymbol{r}) \cdot \nabla \psi+h_{\mathrm{N}}+\phi=\text { constant }, \\
\nabla^{2} \psi=-(\nabla \psi-\boldsymbol{\Omega} \times \boldsymbol{r}) \cdot \frac{\nabla \rho_{0}}{\rho_{0}} .
\end{gathered}
$$

Equation (13) must be solved subject to the boundary condition

$$
\left.(\nabla \psi-\Omega \times r) \cdot \nabla \rho_{0}\right|_{\text {surf }}=0,
$$

where the surface is defined by $\rho_{0}=0$.

Note that in terms of the velocity in the rotating frame, $v$, its divergence is given by equation (8), while its curl is

$$
\nabla \times v=-2 \Omega .
$$

The Bernoulli integral is

$$
\frac{1}{2} v^{2}+h_{\mathrm{N}}+\phi+\phi_{c}=\text { constant }
$$

The curl equation (15) is solved by

$$
\boldsymbol{v}=-\mathbf{\Omega} \times \boldsymbol{r}+\nabla \psi
$$

and we then recover equations (12) and (13) from equations (16) and (8).

Equations (12) and (13) were solved by Bonazzola et al. (1992) for an illustrative case, but they did not give any sequences of models. The first sequences were constructed by Uryū \& Eriguchi (1997), who solved the same equations for equal mass binaries with polytropic equations of state.

\section{IRROTATIONAL FLOW IN GENERAL RELATIVITY}

Just as in Newtonian fluid mechanics, the velocity of a relativistic perfect fluid can be expressed as the gradient of a potential if the vorticity is zero (see, e.g., Landau \& Lifshitz 1959 or Moncrief 1980). Define the relativistic enthalpy by

$$
h=\frac{\rho+P}{\rho_{0}},
$$

where $\rho$ is the total energy density and $\rho_{0}=m_{B} n$ is the rest-mass density. (We use units with $c=G=1$.) Here $n$ is the baryon number density and $m_{B}$ the mean baryon rest mass. Then the relativistic vorticity tensor is defined as

$$
\omega_{\mu v}=P_{\mu}^{\alpha} P^{\beta}{ }_{\nu}\left[\nabla_{\beta}\left(h u_{\alpha}\right)-\nabla_{\alpha}\left(h u_{\beta}\right)\right] .
$$

Here $u^{\mu}$ is the fluid 4-velocity and $P^{\mu}{ }_{v}=\delta^{\mu}{ }_{v}+u^{\mu} u_{v}$ is the projection tensor. There is another definition of relativistic vorticity, without the $h$ in equation (19). Both definitions reduce to the correct Newtonian limit, where $h \rightarrow 1$, but the definition in equation (19) is the one that leads to a natural definition of potential flow.

For a perfect fluid, the Euler equation can be written

$$
u^{\alpha} \nabla_{\alpha}\left(h u_{\mu}\right)+\nabla_{\mu} h=0 .
$$

Equations (19) and (20) yield a simple expression for the vorticity,

$$
\omega_{\mu v}=\nabla_{v}\left(h u_{\mu}\right)-\nabla_{\mu}\left(h u_{v}\right) .
$$

Thus, if the vorticity is zero, then the quantity $h u_{u}$ can be expressed as the gradient of a potential:

$$
h u_{\mu}=\nabla_{\mu} \psi .
$$

The equation of continuity for the baryon density $n$ is

$$
\nabla_{\alpha}\left(n u^{\alpha}\right)=0,
$$


or

$$
\nabla^{\alpha}\left[(n / h) \nabla_{\alpha} \psi\right]=0 .
$$

The equation of state relates $n$ to $h$, and $h$ is found from the normalization condition $u_{\alpha} u^{\alpha}=-1$, which by equation (22) gives

$$
h=\left[-\left(\nabla_{\alpha} \psi\right)\left(\nabla^{\alpha} \psi\right)\right]^{1 / 2} .
$$

Even for flow in a fixed background geometry, equation (24) with equation (25) is in general a nonlinear equation in $\psi$ and its derivatives. We rewrite it as

$$
\nabla^{\alpha} \nabla_{\alpha} \psi=-\left(\nabla^{\alpha} \psi\right) \nabla_{\alpha} \ln (n / h) .
$$

Then one way of solving equation (26) is by iteration, with either $n / h$ or the whole right-hand side determined from the previous iteration. In general, this procedure will have to be iterated with the solution of the Einstein field equations for the metric.

\section{4. $3+1$ DECOMPOSITION}

We make the usual ADM $3+1$ decomposition of the spacetime metric:

$$
d s^{2}=-\alpha^{2} d t^{2}+\gamma_{i j}\left(d x^{i}+\beta^{i} d t\right)\left(d x^{j}+\beta^{j} d t\right),
$$

where $\alpha$ is the lapse, $\beta^{i}$ is the shift, and $\gamma_{i j}$ is the 3-metric on the $t=$ constant time slices. If we let $\vec{n}$ denote the unit normal to these time slices, and $\vec{k}$ the tangent to the $t$-coordinate lines (i.e., $\vec{k}=\partial / \partial t$ in the ADM coordinate system), then the decomposition is equivalent to writing

$$
\vec{k}=\alpha \vec{n}+\vec{\beta}, \quad \vec{n} \cdot \vec{\beta}=0,
$$

and expressing the 3-metric in a general coordinate system as

$$
\gamma_{\mu \nu}=g_{\mu \nu}+n_{\mu} n_{v} .
$$

We will call any object that is orthogonal to $\vec{n}$ spatial.

The spatial (or three-dimensional) covariant derivative $D_{\mu}$ of any tensor is defined by taking the four-dimensional covariant derivative $\nabla_{\mu}$ and then projecting each free index with a $\gamma_{\mu \nu}$ so that the result is completely spatial. Since $D_{\mu} \gamma_{\alpha \beta}=0$, this covariant derivative is compatible with $\gamma_{\alpha \beta}$, and since a compatible covariant derivative is unique, this definition is equivalent to defining $D_{\mu}$ as the covariant derivative with respect to the 3-metric $\gamma_{\alpha \beta}$.

For later reference, we list some standard relations that hold in the $3+1$ decomposition. The derivative of the normal vector is

$$
\nabla_{\mu} n_{v}=-K_{\mu v}-n_{\mu} D_{v} \ln \alpha .
$$

Here $K_{\mu v}$ is the extrinsic curvature tensor, which is spatial. From equation (30) we see that

$$
n^{\mu} \nabla_{\mu} n_{v}=D_{v} \ln \alpha .
$$

The definition of $D_{\mu}$ gives

$$
\nabla_{\mu} f=D_{\mu} f-n_{\mu} n^{v} \nabla_{v} f
$$

where $f$ is any scalar. Similarly, for any spatial vector $\vec{X}$, we have

$$
\nabla_{\mu} X_{v}=D_{\mu} X_{v}-n_{\mu} n^{\lambda} \nabla_{\lambda} X_{v}-K_{\mu \lambda} X^{\lambda} n_{v} .
$$

\section{QUASI-EQUILIBRIUM}

As discussed in the Introduction, it is a good approximation to treat neutron star binaries as essentially equilibrium states, and to neglect the gravitational radiation on orbital timescales. The quasi-equilibrium assumption is implemented mathematically by requiring that the spacetime admit a Killing vector to express the symmetry: a rotation $\Delta \phi$ about the rotation axis is equivalent to a displacement $\Omega \Delta t$, where $\Omega$ is the uniform orbital angular velocity. We write the Killing vector as

$$
\vec{l}=\frac{\partial}{\partial t}+\Omega \frac{\partial}{\partial \phi} \equiv \vec{k}+\Omega \vec{m} .
$$

Here the generator of rotations about the rotation axis of the binary is denoted by $\partial / \partial \phi$, or $\vec{m}$ in an arbitrary coordinate system. By equation (28), we can also write

$$
\vec{l}=\alpha \vec{n}+\vec{B},
$$

where

$$
\vec{B}=\vec{\beta}+\Omega \vec{m}
$$

is the rotating shift vector. It is equivalent to adding $\Omega \times r$ to the original shift. (Note that here $\vec{B}$ is the negative of $\vec{B}$ defined by Bonazzola, Gourgoulhon, \& Marck 1997.) While $\vec{l}$ is not a Killing vector in the exact metric, in the quasi-equilibrium 
approximation Einstein's equations can be consistently approximated to neglect gravitational radiation and admit $\vec{l}$ as a Killing vector, e.g., by the Wilson-Mathews scheme (Wilson \& Mathews 1989; Wilson 1990; Wilson \& Mathews 1995).

We require that $\vec{l}$ be a symmetry generator for the matter fields as well as for the metric. Mathematically, this means that the Lie derivative along $\vec{l}$ of any matter field must vanish (cf. eq. [3]). Note that since $\psi$ is a potential, we must be careful that the symmetry condition is expressed correctly for it. It is not true that

$$
\frac{\partial \psi}{\partial t}+\Omega \frac{\partial \psi}{\partial \phi}=0 \quad \text { (wrong!) }
$$

Rather,

$$
0=\mathscr{L}_{\hat{\imath}}(h \tilde{u})=\mathscr{L}_{\hat{\imath}}(d \psi)=d\left(\mathscr{L}_{\hat{\imath}} \psi\right) .
$$

Here we have used equation (22) in the notation of differential forms, and used the fact that the Lie derivative of a form commutes with the exterior derivative. Thus, as long as $\mathscr{L}_{\vec{\imath}} \psi$ is a constant, the symmetry will be respected. We write

$$
l^{\mu} \nabla_{\mu} \psi=\mathscr{L}_{\vec{\imath}} \psi=\frac{\partial \psi}{\partial t}+\Omega \frac{\partial \psi}{\partial \phi}=-C,
$$

where the minus sign is chosen for later convenience: $C$ is a positive constant.

Another way of seeing why equation (39) holds is to project the Euler equation (20) along $\vec{l}$. Using Killing's equation

$$
\nabla_{\mu} l_{v}+\nabla_{v} l_{\mu}=0
$$

and the fact that $h$ satisfies the symmetry, we can derive the Bernoulli integral

$$
h u_{\mu} l^{\mu}=\text { constant . }
$$

By equation (22), this is equivalent to equation (39). form

One way of implementing equation (39) is to solve equation (26) by separating out the $t$ dependence with a solution of the

$$
\psi=-C t+f(r, \theta, \phi-\Omega t) .
$$

We will proceed instead to derive a coordinate-independent equation that embodies the symmetry.

We will need expressions for the normal derivatives of scalar quantities that satisfy the symmetry. For $\psi$, equations (35) and (39) give

$$
n^{\mu} \nabla_{\mu} \psi=\frac{1}{\alpha}\left(l^{\mu}-B^{\mu}\right) \nabla_{\mu} \psi=-\frac{1}{\alpha}\left(C+B^{\mu} D_{\mu} \psi\right) .
$$

For a scalar $f$ that satisfies $\mathscr{L}_{\vec{l}} f=0$ we have a similar equation with $C=0$ :

$$
n^{\mu} \nabla_{\mu} f=-\frac{1}{\alpha} B^{\mu} D_{\mu} f
$$

\section{6. $3+1$ DECOMPOSITION OF THE POTENTIAL EQUATION}

We can now derive the $3+1$ decomposition of the potential equation (26). First, equations (32) and (43) give

$$
\nabla_{\mu} \psi=D_{\mu} \psi+n_{\mu}\left(C+B^{v} D_{v} \psi\right) / \alpha .
$$

Therefore,

$$
\nabla^{\mu} \nabla_{\mu} \psi=\nabla^{\mu} D_{\mu} \psi+\left(\nabla^{\mu} n_{\mu}\right)\left(C+B^{v} D_{v} \psi\right) / \alpha+n^{\mu} \nabla_{\mu}\left(C+B^{v} D_{v} \psi\right) / \alpha .
$$

Using equation (33) with $X_{v} \rightarrow D_{v} \psi$, we can write the first term on the right-hand side of equation (46) as

$$
\nabla^{\mu} D_{\mu} \psi=D^{\mu} D_{\mu} \psi-n^{\mu} n^{\lambda} \nabla_{\lambda} D_{\mu} \psi=D^{\mu} D_{\mu} \psi-n^{\lambda} \nabla_{\lambda}\left(n^{\mu} D_{\mu} \psi\right)+\left(n^{\lambda} \nabla_{\lambda} n^{\mu}\right) D_{\mu} \psi=D^{\mu} D_{\mu} \psi-0+\left(D_{\mu} \psi\right) D^{\mu} \ln \alpha .
$$

In the second term on the right-hand side of equation (46) we replace $\nabla^{\mu} n_{\mu}$ by $-K\left(=-K^{\mu}{ }_{\mu}\right)$. For the third term, we use equation (44):

$$
n^{\mu} \nabla_{\mu} \frac{1}{\alpha}\left(C+B^{v} D_{v} \psi\right)=-\frac{1}{\alpha} B^{\mu} D_{\mu} \frac{1}{\alpha}\left(C+B^{v} D_{v} \psi\right) .
$$

Substituting equations (47) and (48) in equation (46), we find

$$
\nabla^{\mu} \nabla_{\mu} \psi=D^{\mu} D_{\mu} \psi+\left(D_{\mu} \psi\right) D^{\mu} \ln \alpha-\frac{K}{\alpha}\left(C+B^{v} D_{v} \psi\right)+\frac{1}{\alpha^{3}}\left(B^{\mu} D_{\mu} \alpha\right)\left(C+B^{v} D_{v} \psi\right)-\frac{B^{\mu}}{\alpha^{2}} D_{\mu}\left(B^{v} D_{v} \psi\right)
$$


For the right-hand side of equation (26) we use equation (45) and a similar equation with $\psi$ replaced by $\ln (n / h)$ and $C=0$. So the final form of equation (26) is

$$
D^{i} D_{i} \psi+\left(D_{i} \psi\right) D^{i} \ln \alpha+\left(C+B^{j} D_{j} \psi\right)\left(\frac{B^{i}}{\alpha^{2}} D_{i} \ln \alpha-\frac{K}{\alpha}\right)-\frac{B^{i}}{\alpha^{2}} D_{i}\left(B^{j} D_{j} \psi\right)=-\left[D^{i} \psi-\left(C+B^{j} D_{j} \psi\right) \frac{B^{i}}{\alpha^{2}}\right] D_{i} \ln \left(\frac{n}{h}\right) .
$$

Here we have used latin indices, since the equation is completely spatial, with the metric being the 3-metric $\gamma_{i j}$. For numerical work, it may be advantageous to rewrite equation (50) in an equivalent form. For example, define

$$
\lambda=C+B^{j} D_{j} \psi=\alpha\left[h^{2}+\left(D_{i} \psi\right)^{2}\right]^{1 / 2},
$$

where the second equality follows from equation (57) below. Then equation (50) becomes

$$
D^{i} D_{i} \psi-B^{i} D_{i} \frac{\lambda}{\alpha^{2}}-\frac{\lambda K}{\alpha}=-\left(D^{i} \psi-\frac{\lambda}{\alpha^{2}} B_{i}\right) D_{i} \ln \left(\frac{\alpha n}{h}\right) .
$$

An equivalent form is obtained by the substitution

$$
\alpha K=D_{i} B^{i}
$$

which follows from Killing's equation (eq. [40]). Then

$$
D^{i} D_{i} \psi-D_{i} \frac{\lambda B^{i}}{\alpha^{2}}=-\left(D^{i} \psi-\frac{\lambda}{\alpha^{2}} B_{i}\right) D_{i} \ln \left(\frac{\alpha n}{h}\right) .
$$

Since a consistent approximation scheme for Einstein's equations with the assumptions made here, such as the WilsonMathews scheme, typically has $K=0$, the form (52) may be better.

At the surface of either star, $n \rightarrow 0, h \rightarrow 1$. Thus equation (52) or equation (54) must be solved together with the boundary condition

$$
\left|\left(D^{i} \psi-\frac{\lambda}{\alpha^{2}} B_{i}\right) D_{i} n\right|_{\text {surf }}=0 .
$$

The Bernoulli integral for the matter distribution follows from substituting equation (45) in equation (25):

$$
h^{2}=-\left(D^{i} \psi\right) D_{i} \psi+\frac{1}{\alpha^{2}}\left(C+B^{j} D_{j} \psi\right)^{2},
$$

or, equivalently,

$$
C=-B^{j} D_{j} \psi+\alpha\left[h^{2}+\left(D_{i} \psi\right)^{2}\right]^{1 / 2} .
$$

The positive sign for the square root in equation (57) is determined by the Newtonian limit (see $\S 7$ ).

\section{NEWTONIAN LIMIT}

It is easy to see that the above equations reduce to the expected form in the Newtonian limit. The Newtonian limit of the various quantities is

$$
\begin{gathered}
D_{i} \psi \rightarrow \partial_{i} \psi, \\
B_{i} \rightarrow(\boldsymbol{\Omega} \times \boldsymbol{r})_{i}, \\
\alpha \rightarrow 1+\phi, \\
h \rightarrow 1+h_{\mathrm{N}}, \\
K \rightarrow 0 .
\end{gathered}
$$

Thus equation (57) becomes

$$
C \rightarrow-(\boldsymbol{\Omega} \times \boldsymbol{r})_{i} \partial_{i} \psi+\left[1+\phi+h_{\mathrm{N}}+\frac{1}{2}\left(\partial_{i} \psi\right)^{2}\right] .
$$

In leading order, $C \rightarrow 1$. If we define $C=1+C^{\prime}$, then in next order

$$
C^{\prime}=\phi+h_{\mathrm{N}}+\frac{1}{2}\left(\partial_{i} \psi\right)^{2}-(\boldsymbol{\Omega} \times r)_{i} \partial_{i} \psi .
$$

This is equation (12).

Similarly, neglecting $O\left(v^{2}\right)$ corrections, we see that equation (50) reduces to

$$
\nabla^{2} \psi=-\left[\partial_{i} \psi-(\mathbf{\Omega} \times r)_{i}\right] \frac{\partial_{i} n}{n},
$$

which is equation (13). 


\section{CONCLUSIONS}

Equation (52), together with equation (56) or equation (57), is the principal result of this paper. It is a relatively simple Poisson-like equation for a scalar field that determines the fluid velocity for irrotational flow in the quasi-equilibrium approximation. Bonazzola, Gourgoulhon, \& Marck (1997) have considered the same problem as treated here, but their formalism is considerably more complicated. Their results are compared with ours in Appendix A. We do note, however, that this paper was inspired by reading theirs.

The equations derived here, together with a compatible approximation to Einstein's equations such as the Wilson-Mathews scheme, allow one to construct sequences of neutron star binaries that should be very good approximations to actual binaries during the inspiral phase. The formalism is not much more complicated than that used by Baumgarte et al. (1997a,1997b, 1997c, 1997d) to construct corotating sequences, since equations very similar to equation (50) are already being solved in that case. Work is underway to construct such more realistic sequences.

After this paper was submitted for publication, I became aware of an independent paper by Shibata (1998) treating the same problem. His equations (2.18) and (2.22) are completely equivalent to our equations (57) and (54) when the appropriate substitutions are made.

I thank Larry Kidder for helpful discussions. This work was supported in part by NSF grant PHY 94-08378 to Cornell University, and by the Grand Challenge grant NSF PHY 93-18152/ASC 93-18152.

\section{APPENDIX}

\section{COMPARISON WITH THE FORMALISM OF BONAZZOLA, GOURGOULHON, \& MARCK}

Bonazzola, Gourgoulhon, \& Marck (1997) have developed a different way of treating irrotational quasi-equilibrium binaries. Instead of working directly with the 4-velocity of the fluid as we have, they consider the 3-velocity in the rotating frame. They decompose the 4-velocity as

$$
\vec{u}=\Gamma(\vec{v}+\vec{V}),
$$

where $\vec{v} \propto \vec{l}$ is the 4-velocity of the rotating frame, $\vec{V}$ is the 3-velocity in the rotating frame, and $\Gamma=(1-\vec{V} \cdot \vec{V})^{-1 / 2}$ is the Lorentz factor. Then they derive the Bernoulli integral (eq. [41]), which in their variables is

$$
\ln h+\Phi+\ln \Gamma=\text { constant }
$$

where $\Phi=\ln \left(\alpha^{2}-\vec{B} \cdot \vec{B}\right)^{1 / 2}$, and also equations for the divergence and curl of the velocity field,

$$
\nabla \cdot \vec{V}=-\nabla_{\vec{V}} \ln (n \Gamma), \quad \nabla \wedge \vec{V}=-2 \vec{\omega}+(\nabla \wedge \vec{V}+2 \vec{\omega}) \cdot \vec{V} \frac{\vec{V}}{\vec{V} \cdot \vec{V}}-\vec{V} \wedge \nabla \Phi .
$$

The "cross product" operation $\wedge$ used for the curl is defined in the rest frame of the rotating observer:

$$
(\vec{a} \wedge \vec{b})_{\mu}=v^{\alpha} \epsilon_{\alpha \mu \beta \gamma} a^{\beta} b^{\gamma} .
$$

The "rotation vector" $\vec{\omega}$ is defined by

$$
\nabla \wedge \vec{v}=2 \vec{\omega} .
$$

In the Newtonian limit, where $\vec{\omega} \rightarrow \boldsymbol{\Omega}$, these equations reduce to equations (8), (15), and (16).

Bonazzola, Gourgoulhon, \& Marck (1997) carry out the $3+1$ decomposition of the above equations to get threedimensional equations for the velocity field. They then decompose the velocity using both scalar and vector potentials, and obtain a scalar and a vector Poisson-like equation. In the Newtonian limit, the vector potential has an analytic solution corresponding to the term $-\boldsymbol{\Omega} \times \boldsymbol{r}$ in equation (17), leaving just the scalar potential to be solved for. However, there does not seem to be an analytic solution for the vector potential in the relativistic case, and so one has to solve for it numerically as well.

The procedure of Bonazzola, Gourgoulhon, \& Marck (1997) can actually be simplified somewhat by working with a slightly different three-dimensional velocity $\vec{p}$, defined by

$$
\vec{p}=e^{-\Phi} \vec{V}
$$

Then equations (68) become

$$
\nabla \cdot \vec{p}=-\nabla_{\vec{p}} \ln \left(n \Gamma e^{\Phi}\right), \quad \nabla \wedge \vec{p}=-2 e^{-\Phi} \vec{\omega} .
$$

However, after the $3+1$ decomposition and introduction of scalar and vector potentials, one is still left with complicated equations.

Abramovici, A., et al. 1992, Science, 256, 325

Baumgarte, T. W., Shapiro, S. L., Cook, G. B., Scheel, M. A., \& Teukolsky,

S. A. 1997a, in Proc. 18th Texas Symp. on Relativistic Astrophysics, ed.

A. Olinto, J. Friedman, \& D. Schramm (Singapore: World Scientific) 1997b, Phys. Rev. Lett., 79, 1182

1997c, preprint (gr-qc/9709026)

. 1997d, preprint (gr-qc/9705023)

\section{REFERENCES}

Bildsten, L., \& Cutler, C. 1992, ApJ, 400, 175

Bonazzola, S., Gourgoulhon, E., Haensel, P., \& Marck, J.-A. 1992, in Approaches to Numerical Relativity, ed. R. d'Inverno (Cambridge: Cambridge Univ. Press), 230

Bonazzola, S., Gourgoulhon, E., \& Marck, J.-A. 1997, Phys. Rev. D, 56, 7740

Cook, G. B., Shapiro, S. L., \& Teukolsky, S. A. 1996, Phys. Rev. D, 53, 5533 
Eichler, D., Livio, M., Piran, T., \& Schramm, D. N. 1989, Nature, 340, 126

Hulse, R. A., \& Taylor, J. H. 1975, ApJ, 195, L51

Kochanek, C. S. 1992, ApJ, 398, 234

Lai, D. 1994, MNRAS, 270,611

Landau, L. D., \& Lifshitz, E. M. 1959, Fluid Mechanics (Reading: Addison-Wesley), 504

Mathews, G. J., Marronetti, P., \& Wilson, J. R. 1997, preprint (gr-qc/ 9710140)

Moncrief, V. 1980, ApJ, 235, 1038

Narayan, R., Paczyński, B., \& Piran, T. 1992, ApJ, 395, L83

Paczyński, B. 1986, ApJ, 308, L51

Shibata, M. 1998, preprint (gr-qc/9803085)
Thorne, K. S. 1994, in Relativistic Cosmology, Proc. Eighth NishinomiyaYukawa Memorial Symp., ed. M. Sasaki (Tokyo: Universal Academy Press), 67

Uryū, K., \& Eriguchi, Y. 1997, MNRAS, submitted (astro-ph/9712203)

Wilson, J. R. 1990, in Texas Symp. on 3-Dimensional Numerical Relativity, ed. R. A. Matzner (Austin: Univ. Texas)

Wilson, J. R., \& Mathews, G. J. 1989, in Frontiers in Numerical Relativity, ed. C. R. Evans, L. S. Finn, \& D. W. Hobill (Cambridge: Cambridge Univ. Press), 306

1995, Phys. Rev. Lett., 75, 4161

Wilson, J. R., Mathews, G. J., \& Marronetti, P. 1996, Phys. Rev. D, 54, 1317 